\title{
DEFORMATION THEORY AND THE TAME FUNDAMENTAL GROUP
}

\author{
BY \\ DAVID HARBATER ${ }^{1}$
}

\begin{abstract}
Let $U$ be a curve of genus $g$ with $n+1$ points deleted, defined over an algebraically closed field of characteristic $p>0$. Then there exists a bijection between the Galois finite étale covers of $U$ having degree prime to $p$, and the finite $p^{\prime}$-groups on $n+2 g$ generators. This fact has been proven using analytic considerations; here we construct such a bijection algebraically. We do this by algebraizing an analytic construction of covers which uses Hurwitz families. The process of algebraization relies on a deformation theorem, which we prove using Artin's Algebraization Theorem, and which allows the patching of local families into global families. That our construction provides the desired bijection is afterwards verified analytically.
\end{abstract}

1. Introduction. The fundamental group $\pi_{1}(U)$ of an algebraic curve $U$ over an algebraically closed field $k$ has been studied by Grothendieck, by means of specialization to curves over C. His results [7, XIII, 2.12] can be viewed as a classification of the Galois finite étale covers of the curve $U$, in the case of characteristic 0 , and of the $p^{\prime}$-Galois covers, in the case of characteristic $p$. Inasmuch as the proof relies on a reduction to the analytic category, where covering spaces are classified with the aid of paths, the proof does not give

(i) an algebraic description of the finite étale covers of $U$, or

(ii) information about the étale covers of $U$ whose corresponding ramified covers of $X$ have wild ramification.

In this paper the first of these two points will be addressed, by constructing tame covers using algebraic deformation theory (perhaps an obvious tool when dealing with homotopy!). In a subsequent paper a parallel approach to wild covers will be used to address the second point.

$\$ 2$ describes a cut-and-paste construction which yields arbitrary Galois covers of a Riemann surface. $\$ 3$ begins the process of algebraizing this construction, using the notion of mock cover, an object arising in $\$ 2$. $\$ 4$ proves a deformation theorem, allowing the patching of local families of covers into global families. $\S 5$ applies this theorem to the ideas of $\S 3$, completing the algebraization process over $\mathbf{C}$, and then generalizing it to other algebraically closed fields. A possible application to moduli

Received by the editors May 10, 1979 and, in revised form, December 7, 1979.

AMS (MOS) subject classifications (1970). Primary 14D15, 14H30; Secondary 14D20, 16A58, 32 G15.

Key words and phrases. Fundamental group, étale cover, deformation, Hurwitz family, mock cover, algebraization.

${ }^{1}$ This research was supported by an American Mathematical Society Research Fellowship, and by NSF Grant MCS-7824169. 
spaces of curves is also described. $\$ 6$ gives an example of the construction of covers.

This paper was largely adapted from part of the author's M.I.T. doctoral thesis [8]. The author wishes to thank his thesis advisor, Michael Artin, for his advice and encouragement. He also wishes to thank Michael Fried for his careful reading of and comments upon the thesis.

2. A topological construction. Let $X$ be a Riemann surface of genus $g, L=$ $\left\{x_{0}, \ldots, x_{n}\right\}$ a set of points in $X$, and $U=X-L$. If $u$ is a point of $U$, then it is well known that there is a set of loops $B=\left\{\alpha_{0}, \ldots, \alpha_{n+2 g}\right\}$ at $u$ in $U$ whose homotopy classes $a_{j}$ generate $\pi_{1}(U, u)$ and satisfy the single relation

$$
\left(\prod_{j=0}^{n} a_{j}\right)\left(\prod_{j=1}^{g} a_{n+j} a_{n+g+j} a_{n+j}^{-1} a_{n+g+j}^{-1}\right)=1 .
$$

Indeed $B$ may be chosen so that $\alpha_{j}(j \leqslant n)$ winds once around $x_{j}$, counterclockwise (relative to the orientation given by the complex structure) and around no other $x_{i}$; and so that $\alpha_{n+j}$ and $\alpha_{n+g+j}(1 \leqslant j \leqslant g)$ wind once around the " $j$ th hole". We call such a choice of loops a standard homotopy basis for $(U, u)$. Relative to $B$, there are one-to-one correspondences between

2.2 (i) Isomorphism classes of pointed Galois covers of $(U, u)$, with a fixed action of $G$ as Galois group;

(ii) epimorphisms $\varphi: \pi_{1}(U, u) \rightarrow G$;

(iii) elements $d=\left(d_{0}, \ldots, d_{n+2 g}\right) \in G^{n+2 g+1}$ whose entries generate $G$ and satisfy (2.1).

The bijection (i) $\leftrightarrow$ (ii) is given by the Galois theory of covers, as every cover is a quotient of the universal cover; (ii) $\leftrightarrow$ (iii) follows from the description above of $\pi_{1}(U)$, via generators $a_{j}$ and the single relation (2.1).

Given a cover $(V, v) \rightarrow(U, u)$ as in (i), the corresponding element $d$ in (iii) can be described without reference to the universal cover. Namely, each loop $\alpha_{j}$ in $B$ lifts to a unique path $\alpha_{j}^{*}$ in $V$ having initial point $v$, and there is a unique element $d_{j}$ in $G$ sending $v$ to the final point of $\alpha_{j}^{*}$. The element $d$ is then $\left(d_{0}, \ldots, d_{n+2 g}\right)$.

The goal of this section is to describe similarly the inverse correspondence (iii) $\rightarrow$ (i); that is, to construct, without reference to the universal cover, the $G$-cover corresponding to $d=\left(d_{0}, \ldots, d_{n+2 g}\right)$. Actually, we phrase this instead in terms of

(iv) Elements $\left(d_{1}, \ldots, d_{n+2 g}\right)$ of $G^{n+2 g}$ whose entries generate $G$.

This is equivalent to 2.2(iii), provided that $L \neq \varnothing$. Since an unramified cover of $U$ is also such a cover of $U-\left\{x_{0}\right\}$, for $x_{0}$ arbitrary, we shall assume that $L \neq \varnothing$. Henceforth the corresponding element of (iv) will be called the data of the cover.

2.3. Our construction of covers relies on work of Hurwitz [9]; cf. also [5]. Fix an integer $r$, and let $\Delta \subset X^{r}$ be the weak diagonal, consisting of $r$-tuples with two equal entries. Define the Hurwitz space $H_{r}$ to be the set of isomorphism classes of pairs $(Z \rightarrow X, x)$, where $x=\left(x_{1}, \ldots, x_{r}\right) \in X^{r}-\Delta$ and $Z \rightarrow X$ is a ramified cover with branch locus $\left\{x_{1}, \ldots, x_{r}\right\}$. The projection $H_{r} \rightarrow X^{r}-\Delta$ becomes a covering map under a natural topology for $H_{r}$. The Hurwitz space may be completed to a ramified cover $\bar{H}_{r} \rightarrow X^{r}$, and for some (ramified) cover $\stackrel{f}{\rightarrow} \bar{H}_{r}$, 
there is a Hurwitz family $W \rightarrow X \times \bar{H}$ whose fibre over each $h \in H=f^{-1}\left(H_{r}\right)$ is a ramified cover of $X$ lying in the isomorphism class $f(h)$. The fibres of $W$ over $\bar{H}-H$ arise as a result of branch points coalescing.

While Hurwitz's original construction relied on the analytic topology of $X$, Fulton [5] has shown how it can be made algebraic. His Theorem 6.3 algebraically constructs the components of $W$ corresponding to "simple covers", i.e. $N$-sheeted covers with at least $N-1$ points in each fibre. A modification yields the component having any given cover as a fibre.

For a more detailed study of Hurwitz families, with an eye toward arithmetic questions, see M. Fried's papers [3] and [4].

2.4. Let $G$ be a group of order $N$, and $d=\left(d_{1}, \ldots, d_{r}\right) \in G^{r}$. Choose points $x_{1}, \ldots, x_{r}$ in $X$, and disjoint open discs $B_{1}, \ldots, B_{r}$ containing them. For each $j$ let $\sigma_{j}$ be an arc (i.e. non-self-intersecting path) in $B_{j}$ from $x_{j}$ to another point $y_{j}$. A ramified cover $Z \rightarrow X$ may be defined by taking $N$ copies of $X$, indexed by the elements of $G$, "cutting" along the copies of the $\sigma_{j}$ 's, and "pasting" the right side of $\sigma_{j}$ on $X_{g}$ (relative to the parametrization of $\sigma_{j}$ and the orientation of $X$ ) to the left side of $\sigma_{j}$ on $X_{g d_{j}}$. Call $Z \rightarrow X$ the slit cover of $(X ; x ; y)$ with data $d$; it is independent of the choice of the $\sigma_{j}$. This cover is provided with a $G$-action, such that $h \in G$ sends $X_{g}$ to $X_{h g}$. It thus becomes a principal (ramified) $G$-cover, i.e. $G$ acts simply transitively on the generic fibre. It is Galois with group $G$ if and only if the $d_{j}$ 's generate $G$.

In particular, let $d \in G^{n+2 g}$ be as in 2.2(iv), under the hypotheses given there. Let $x_{n+1}, \ldots, x_{n+2 g}$ be distinct points of $U-\{u\}$, write $x=\left(x_{1}, \ldots, x_{n+2 g}\right)$, and let $Z \rightarrow X$ be the slit cover of $(X ; x ; y)$ with data $d$ (for some choice of $y \in$ $\left.X^{n+2 g}\right)$. The isomorphism class of $Z \rightarrow X$ is a point in the fibre of $H_{2 n+4 g} \rightarrow$ $X^{2 n+4 g}$ over $\left(x_{1}, \ldots, x_{n}\right) \times X^{n+4 g}$; let $H$ be its connected component in this fibre. By a variant on the terminology of $\$ 2.3$, we shall call $H \rightarrow X^{n+48}$ the Hurwitz space for $(X ; x)$ with data $d$; it is independent of $y$. As in $\S 2.3, H$ gives rise to a family of covers $\bar{W} \rightarrow X \times \bar{H}$, which we shall call a Hurwitz family for $(X ; x)$ with data $d$.

The correspondence 2.2 (iv) $\rightarrow$ (i) can now be given:

Proposition 2.5. Let $\bar{W} \rightarrow X \times \bar{H}$ be a Hurwitz family for $(X ; x)$ with data $d$. There is a point $h \in H$ over $\left(x_{0}, \ldots, x_{0}\right) \in X^{n+4 g}$ such that the fibre of $\bar{W}$ over $U \times\{h\}$ is the cover of $U$ with data $d$.

Proof. If $X=\mathbf{P}^{1}$, then choose disjoint arcs (or "slits") $\sigma_{1}, \ldots, \sigma_{n}$ from $x_{1}, \ldots, x_{n}$ to $x_{0}$. These may be taken so that $\sigma_{j}$ intersects no member of the standard homotopy basis $B$ except for $\alpha_{0}$ and $\alpha_{j}$, each of which it meets once. Choose $\varepsilon$ so that for all $j, y_{j}=\sigma_{j}(\varepsilon)$ is inside the loop $\alpha_{j}$ (i.e. is in the component of $x_{j}$ in the complement of the loop). Thus $\bar{\sigma}=\left(\bar{\sigma}_{1}, \ldots, \bar{\sigma}_{n}\right)$ is a path in $X^{n}$ from $y=\left(y_{1}, \ldots, y_{n}\right)$ to $\left(x_{0}, \ldots, x_{0}\right)$, where $\bar{\sigma}_{j}=\sigma_{j} \mid[\varepsilon, 1]$. Let $\sigma^{*}$ be the unique lifting of $\bar{\sigma}$ to $\bar{H}$ whose initial point corresponds to the slit cover of $(X ; x ; y)$ with data $d$. Its final point corresponds to the cover obtained by making a slit cover construction using $\sigma_{1}, \ldots, \sigma_{n}$. This cover has branch locus $L=\left\{x_{0}, \ldots, x_{n}\right\}$, and it is easily verified that it has data $d$ relative to $B$. 
For a more general Riemann surface $X$ of genus $g$, augment $\sigma_{1}, \ldots, \sigma_{n}$ by loops $\sigma_{n+1}, \ldots, \sigma_{n+2 g}$ at $x_{0}$. Choose these so that $\sigma_{j}($ for $j>n$ ) intersects no members of $B$ except for $\alpha_{0}$, which it crosses twice, and $\alpha_{j}$, which it crosses once from left to right. (Cf. Figure 2.6, for the case $g=1, n=2$.) For each $j>n$ choose distinct points $x_{j}=\sigma_{j}(t)$ and $y_{j}=\sigma_{j}\left(t+\varepsilon^{\prime}\right)$ outside the loop $\alpha_{0}$ and off $\alpha_{j}$. Let $\bar{\sigma}_{j}$ be as before for $j \leqslant n$; for $j>n$ let $\bar{\sigma}_{j}=\sigma_{j} \mid\left[t+\varepsilon^{\prime}, 1\right]$ and let $\sigma_{j}^{\prime}=\left(\sigma_{j} \mid[0, t]\right)^{-1}$. Reasoning as for $\mathbf{P}^{1}$, these paths in $X$ define a path in $\bar{H}$ whose initial point corresponds to the slit cover of $(X ; x ; y)$ with data $d$, and whose final point has branch locus $\left\{x_{0}, \ldots, x_{n}\right\}$ and data $d$ relative to $B$.

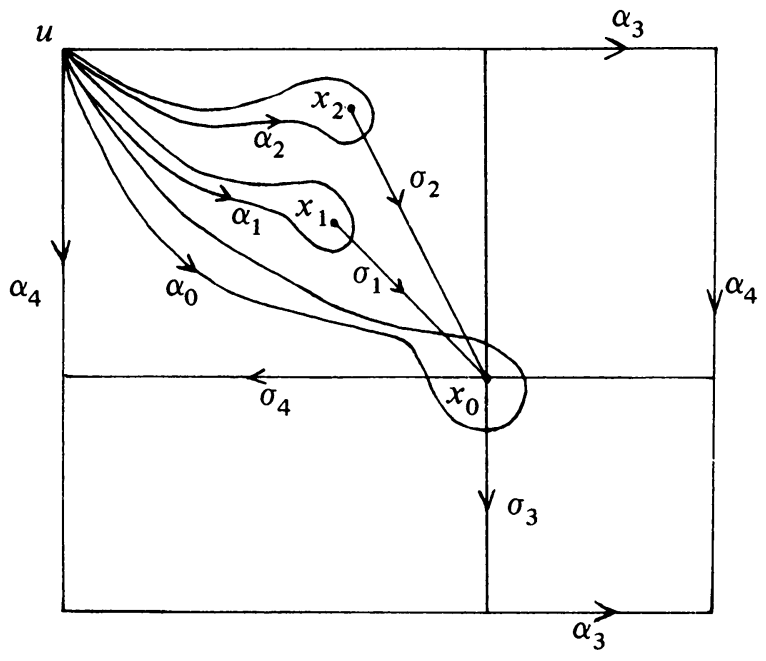

FiguRE 2.6

2.7. If a slit cover with given data could be constructed algebraically, then so could a corresponding Hurwitz family (cf. \$2.3), and hence a cover having given data-thus algebraizing 2.2(iv) $\rightarrow$ (i). But as the notion of slit is not an algebraic one, we rely instead on another, simpler fibre of the Hurwitz family. Namely, in the above Hurwitz family, there is a fibre which is a mock cover of $X$; i.e. it is a union of "sheets", each homeomorphic to $X$, and meeting over the branch points. (The mock cover corresponds to the point $h_{0}$ of $\bar{H}$ which lies over $s_{0}=$ $\left(x_{n+1}, \ldots, x_{n+2 g}, x_{1}, \ldots, x_{n+2 g}\right)$ and is "near" the point corresponding to the slit cover.)

Observe that the sheets of the mock cover $Z_{0} \rightarrow X$ can be labelled by the elements of $G$ such that two sheets meet over $x_{j}$ if and only if their labels are in the same right coset of $d_{j}$. Such a mock cover we call a mock cover of $(X ; x)$ with data d.

Mock covers form the key to the problem of algebraizing Hurwitz families with given data, and we begin with them in $\$ 3$.

3. Construction of mock covers. Let $k$ be a field or a complete discrete valuation ring of characteristics $p \geqslant 0$, containing $m$ th roots of unity $\zeta_{m}$ for all $m$ prime to $p$. The $\zeta_{m}$ 's may be taken to be compatible, i.e. $\zeta_{m n}^{n}=\zeta_{m}$. Consider a connected 
smooth curve $X$ over $k$, and let $L$ be a finite subset of $X$. By a cover of $X$ with branch locus $L$ is meant a finite morphism $Z \stackrel{\pi}{\rightarrow} X$ which is étale over $U=X-L$. Following \$2.7, we say that such a cover is a mock cover if the restriction of $\pi$ to each irreducible component (or sheet) is an isomorphism.

EXAmple 3.1. Take $X=\mathbf{A}^{1}=\operatorname{Spec} k[x]$ and $L=\{0\}$. Let

$$
E_{m}=k[x, z] /\left(z^{m}-x^{m}\right)
$$

and let $Z=\operatorname{Spec} E_{m}$. Then $Z \rightarrow \mathbf{A}^{1}$ is an $m$-sheeted mock cover with branch locus $L$. It is acted upon by $Z / m$, whose generator $g$ operates by

$$
z \leadsto \zeta_{m} z \text {. }
$$

This action permutes the sheets cyclically, sending the $i$ th sheet

$$
Z_{g^{i}}: z=\zeta^{i} x
$$

to the $(i+1)$ st sheet, $i \in \mathbf{Z} / m$. In particular, if $k=\mathbf{C}$, then in the terminology of $\S 2.7, Z \rightarrow \mathbf{A}^{1}$ is a mock cover of $\left(\mathbf{A}^{1} ; 0\right)$ with data $g \in \mathbf{Z} / m$, under the analytic topology.

More generally, let $X$ be any curve, and $x_{0} \in X$. There is an open neighborhood $U_{0}$ of $x_{0}$ and an étale morphism $\varphi: U_{0} \rightarrow \mathbf{A}^{1}$ such that $x_{0}=\varphi^{-1}(0)$. Call such a $\varphi$ a local coordinate at $x_{0}$. Let $Z_{0} \rightarrow U_{0}$ be the pullback under $\varphi$ of the above mock cover $Z \rightarrow \mathbf{A}^{1}$. This is a $\mathbf{Z} / m$-mock cover of $U_{0}$ with branch locus $\left\{x_{0}\right\}$. Over any $k$, call such a cover a standard mock cover of $\left(U_{0}, x_{0}\right)$ with data $g: z \leadsto \zeta z$.

Proposition 3.2. Standard mock covers are Galois with cyclic Galois group, and are independent of the choice of local coordinate.

Proof. Letting $E_{m}$ be as in Example 3.1, there is an inclusion

$$
\alpha: E_{m} \hookrightarrow k[x, u] /\left(u^{m}-1\right)
$$

defined by $z \leadsto u x, x \leadsto x$. There is also an isomorphism

$$
I: k[x, u] /\left(u^{m}-1\right) \stackrel{\sim}{\rightarrow} k[x]^{m}=k[x] \times \cdots \times k[x]
$$

defined by

$$
f(x, u) \rightsquigarrow\left(f(x, 1), f(x, \zeta), \ldots, f\left(x, \zeta^{m-1}\right)\right),
$$

with inverse $J$ given by the Fourier inversion formula:

$$
\left(f_{0}(x), \ldots, f_{m-1}(x)\right) \leadsto \sum_{i=0}^{m-1}\left[\frac{1}{m} \sum_{j=0}^{m-1} \zeta^{-i j} f_{j}(x)\right] u^{i} .
$$

Thus

$$
\begin{aligned}
E_{m} & \approx J^{-1} \circ \alpha\left(E_{m}\right) \\
& =\left\{\left(f_{0}, \ldots, f_{m-1}\right) \in k[x]^{m}: x^{i} \mid \sum_{j=0}^{m-1} \zeta^{-i j} f_{j} \text { for } 1 \leqslant i \leqslant m\right\} .
\end{aligned}
$$

Under this isomorphism, $z \in E_{m}$ corresponds to the $m$-tuple $\left(x, \zeta x, \ldots, \zeta^{m-1} x\right)$.

Now if $Z_{0}=\operatorname{Spec} S \rightarrow U_{0}=\operatorname{Spec} R$ is an arbitrary standard mock cover of degree $m$, and if the branch point $x_{0}$ corresponds to the maximal ideal $m \subset R$, then 
we may identify

$$
S=\left\{\left(f_{0}, \ldots, f_{m-1}\right) \in R^{m}: \sum \zeta^{-i j} f_{j} \in \mathfrak{m}^{i} \text { for } 1 \leqslant i \leqslant m\right\} .
$$

This proves the second assertion.

For the first assertion, observe that

$$
\mathrm{Gal} Z_{0} \hookrightarrow \mathrm{Gal} X_{0}^{\amalg m} \approx S_{m},
$$

the symmetric group on $\{0,1, \ldots, m-1\}$. Here the action of $\theta \in S_{m}$ is given on the ring level by

$$
\left(f_{0}, \ldots, f_{m-1}\right) \leadsto\left(f_{\theta(0)}, \ldots, f_{\theta(m-1)}\right) .
$$

The generator of $\mathrm{Z} / m \subset \mathrm{Gal} Z_{0}$ corresponds to the cyclic permutation $(0,1, \ldots, m-1) \in S_{m}$, and we are reduced to showing that every element of Gal $Z_{0}$ corresponds to a power of this cycle.

The element $x \in k[x] \subset R$ lies in $m-m^{2}$, and the $m$-tuple $\left(x, \zeta x, \ldots, \zeta^{m-1} x\right)$ is in the ring $S$. If a permutation $\theta \in S_{m}$ belongs to the Galois group, then

$$
\left(\zeta^{\theta(0)} x, \zeta^{\theta(1)} x, \ldots, \zeta^{\theta(m-1)} x\right) \in S .
$$

Thus $\sum_{j=0}^{m-1} \zeta^{-i j+\theta(j)} x$ lies in $\mathrm{m}^{i}$, hence equals 0 for $i>1$. The result now follows from the

ClaIM. If $\theta \in S_{m}$ and $\sum_{j=0}^{m-1} \zeta_{m}^{-i j+\theta(j)}=0$ for all $1<i<m$, then $\theta$ is a power of the cycle $(0,1, \ldots, m-1)$.

Proof of Claim. The group $G$ generated by $\zeta$ is cyclic of order $m$, and its characters are

$$
\chi_{i}: \zeta^{j} \leadsto \zeta^{i j}, \quad i=0, \ldots, m-1 .
$$

The function $T: G \rightarrow k$ defined by $T\left(\zeta^{j}\right)=\zeta^{\theta(j)}$ is a class function, as $G$ is abelian. Now $T$ is orthogonal to $\chi_{0}$ since $\theta$ is a permutation, and $T$ is orthogonal to $\chi_{2}, \ldots, \chi_{m-1}$ by hypothesis. Thus $T=\lambda \chi_{1}$ for some $\lambda$. So $\lambda=T(1)=\zeta^{j_{0}}$ for some fixed $j_{0}$, or in other words

$$
\theta(j) \equiv j+j_{0} \quad(\bmod m) .
$$

This proves the claim, and the proposition.

As a consequence of Proposition 3.2, the data $g \in \mathrm{Gal}_{U_{0}} Z_{0}$ is well defined on the isomorphism class of a standard mock cover $Z_{0} \rightarrow U_{0}$.

3.3. Let $X$ be a curve over $k, L=\left\{x_{1}, \ldots, x_{r}\right\}$, and $U=X-L$. Let $Z \rightarrow X$ be a principal $G$-mock cover with branch locus $L$. Its sheets may be labelled by the elements of $G$ so that $h \in G$ sends $Z_{g}$ to $Z_{h g}$; here the "identity sheet" may be chosen arbitrarily. Let $x_{j}^{*}$ be the point on $Z_{e}$ over $x_{j}$, and for each $j$ choose an open neighborhood $U_{j}$ of $x_{j}$ which contains no other $x_{i}$. The fibre of $Z$ over $U_{j}$ is a $G$-mock cover branched only at $x_{j}$; consider its connected component containing $x_{j}^{*}$. This is an $I_{j}$-mock cover, where $I_{j} \subset G$ is the inertial subgroup of $x_{j}^{*}$. Suppose that it is in fact a standard mock cover (so that, in particular, $I_{j}$ is cyclic), say with data $d_{j} \in I_{j}$. If this is true for all $j$, call $Z \rightarrow X$ a locally standard mock cover of $(X ; x)$ with data $d=\left(d_{1}, \ldots, d_{r}\right) \in G^{r}$. If $k=\mathbf{C}$, then under the analytic topology, $Z \rightarrow X$ is an analytic mock cover of $(X ; x)$ with data $d$ (cf. §2.7). 
Proposition 3.4. Let $X$ be a curve over $k, x=\left(x_{1}, \ldots, x_{r}\right)$ an $r$-tuple of points of $X, G$ a group of order $N$, and $d_{1}, \ldots, d_{r}$ elements of $G$ of orders prime to $p$. Then there is a locally standard $G$-mock cover of $(X ; x)$ with data $d=\left(d_{1}, \ldots, d_{r}\right)$, and it is connected if and only if the $d_{j}$ 's generate $G$.

Proof. Let $E_{m}$ be as in Example 3.1. There is an inclusion of $k[x]$-algebras

$$
\psi_{m}: E_{m} \rightarrow k[x]^{m}
$$

given by the composition of the inclusion

$$
E_{m} \rightarrow k[x, u] /\left(u^{m}-1\right) ; \quad z \leadsto u x,
$$

with the isomorphism

$$
\begin{gathered}
k[x, u] /\left(u^{m}-1\right) \rightarrow k[x]^{m} ; \\
f(x, u) \leadsto\left(f(x, 1), f(x, \zeta), \ldots, f\left(x, \zeta^{m-1}\right)\right) .
\end{gathered}
$$

The image is acted upon by $\mathbf{Z} / m$ via cyclic permutations of the coordinates.

Now for $0 \leqslant j \leqslant r$, let $m_{j}$ be the order of $d_{j}$. For each $g \in G$, the inclusion

$$
\mathbf{Z} / m_{j} \hookrightarrow G ; \quad i \leadsto g d_{j}^{i},
$$

induces, via extension by 0 , an inclusion of $k[x]$-algebras

$$
I_{g}: k[x]^{m_{j}} \rightarrow k[x]^{G}
$$

where $k[x]^{G} \approx k[x]^{N}$ is the algebra of maps $G \rightarrow k[x]$. Let $E_{d_{j}}$ be the subalgebra of $k[x]^{G}$ generated by the images of $\psi_{m}\left(E_{m}\right)$ under the $I_{g}$ 's, as $g$ ranges over a set of left coset representatives of the subgroup generated by $d_{j}$. Then Spec $E_{d_{j}}$ is a (disconnected) $G$-mock cover of $\left(\mathbf{A}^{1}, 0\right)$ with data $d_{j}$. The action of any $g \in G$ on the cover is given by permutation of the coordinates according to left multiplication by $g$.

Next, for each $j$, choose a Zariski open neighborhood $U_{j}=\operatorname{Spec} R_{j}$ of $x_{j}$ in $X$ and a local coordinate $\varphi_{j}: U_{j} \rightarrow \mathbf{A}^{1}$, as in 3.1. Write $U=X-L$, where $L=$ $\left\{x_{1}, \ldots, x_{r}\right\}$. Let $Q$ be the unique coherent sheaf of $\Theta_{X}$-algebras on $X$ satisfying

(i) $\mathbb{Q}\left(U_{j}\right)=\varphi_{j}^{*}\left(E_{d j}\right) \subset\left(R_{j}\right)^{G}$;

(ii) $\mathcal{Q}(V)=R^{G}$ for $V=\operatorname{Spec} R \subset U$;

(iii) the restriction map from $\mathcal{Q}\left(U_{j}\right)$ to $\mathcal{Q}\left(U_{j}-\left\{x_{j}\right\}\right)$ is given by the natural inclusion.

Let $Z=$ Spec $_{\hat{\theta}_{X}} Q$. Since $Q$ is a subsheaf of $\vartheta_{X}^{G}, Z$ is dominated by $X^{\amalg G}$, and thus is a mock cover. Since $g \in G$ acts over every patch by permuting the sheets according to left multiplication by $g$, this action is also defined globally. The data for the Spec $E_{d_{j}}$ 's show that $Z \rightarrow X$ has the correct data. Finally, $Z \rightarrow X$ is connected if and only if the $d_{j}$ 's generate $G$, since sheet $g$ intersects precisely the sheets of the form $g d_{j}^{i}$.

For examples of locally standard mock covers see $\S 6$ of this paper, and $\S 3.5$ of [8].

3.5. We wish to deform a mock cover having prescribed data into an algebraic family of covers which, in the case $k=\mathrm{C}$, is an analytic Hurwitz family with that data. Let $X$ be a curve of genus $g, x=\left(x_{1}, \ldots, x_{n+2 g}\right) \in X^{n+2 g}-\Delta$, and write 
$s=\left(x_{n+1}, \ldots, x_{n+2 g}, x_{1}, \ldots, x_{n+2 g}\right)$. Let $G$ be a group of order $N$, and suppose $d \in G^{n+2 g}$ is tame-i.e. its entries $d_{j}$ are of orders $m_{j}$ prime to the characteristic. The problem is then to find a quasi-finite generically étale morphism of pointed schemes $\left(H, h_{0}\right) \stackrel{\pi}{\rightarrow}\left(X^{n+4 g}, s\right)$ and a $G$-cover $W \stackrel{f}{\rightarrow} X \times H$ such that

3.6. (i) the fibre of $W$ over $h_{0}$ is a mock cover of $(X ; x)$ with data $d$, and

(ii) the fibre of $W$ over any point $h \in H$ is a cover of $X$ branched at $x_{1}, \ldots, x_{n}$ and at the coordinates of $\pi(h)$.

EXAMPLE 3.7. (a) $Z \rightarrow \mathbf{A}^{1}$ be the standard $m$-cyclic mock cover of Example 3.1. This may be completed to a standard mock cover $\bar{Z}$ of $\left(\mathbf{P}^{1}, x=0\right)$ with the same data, by taking the normalization of $\mathbf{P}^{1}$ in $Z$. Now define a family $W$ of covers of $\mathbf{P}^{1}$, parametrized by the $t$-line, given (over $\mathbf{A}^{1}$ ) by

$$
z^{m}=x(x-t)^{m-1}
$$

with group action $g: z \leadsto \zeta_{m} z$. This family satisfies conditions (i) and (ii), with $H=X=\mathbf{P}^{1}$. If $k=\mathbf{C}$, then its normalization is, under the analytic topology, a $\mathbf{Z} / m$-Hurwitz family for $\left(\mathbf{P}^{1}, x=0\right)$ with data $g$. To see this it suffices to show that the fibre $Z_{t_{0}}$ over $t=t_{0}$ is a slit cover with data $g$. This follows from the fact that a counterclockwise loop around $x=0$ of radius $r<t_{0}$ (resp. $r>t_{0}$ ) lifts to a path from $\left(x_{1}, z_{1}\right)$ to $\left(x_{1}, \zeta z_{1}\right)$ (resp. to $\left.\left(x_{1}, z_{1}\right)\right)$.

(b) Slightly more generally, let $G$ be a group of order $N$ and $g \in G$ of order $m$. Relative to the local coordinate $x$, the locally standard $G$-mock cover $Z^{\prime}$ of $\left(\mathbf{P}^{1}, x=0\right)$ with data $g$ is a disjoint union of $N / m$ copies of the $\mathbf{Z} / m$-mock cover $\bar{Z} \rightarrow \mathbf{P}^{1}$ in part (a). The disjoint union $W^{\prime}$ of $N / m$ copies of the $\mathbf{Z} / m$-family $W$ (of part (a)) contains $Z^{\prime}$ as the fibre over $t=0$, and inherits its $G$-action. Reasoning as in (a), the normalization of $W^{\prime}$ is, in the case $k=\mathbf{C}$, an analytic Hurwitz family with group $G$ and data $g$.

For a more general $X$, take a system of local coordinates $\varphi_{j}: U_{j} \rightarrow \mathbf{A}^{1}$ at $x_{1}, \ldots, x_{n+2 g}$, as in the proof of 3.4. Let

$$
S=U_{n+1} \times \cdots \times U_{n+2 g} \times U_{1} \times \cdots \times U_{n+2 g} \subset X^{n+4 g}
$$

and let

$$
\Phi: \varphi_{n+1} \times \cdots \times \varphi_{n+2 g} \times \varphi_{1} \times \cdots \times \varphi_{n+2 g}: S \rightarrow A^{n+4 g},
$$

where we regard $\mathbf{A}^{n+4 g}=\operatorname{Spec} A$,

$$
A=k\left[\tau_{n+1}, \ldots, \tau_{n+2 g}, t_{1}, \ldots, t_{n+2 g}\right] .
$$

We then make the following

Definition 3.8. Let $W \rightarrow X \times H$ be a $G$-cover and $\left(H, h_{0}\right) \rightarrow\left(X^{n+4 g}, s\right)$ a morphism satisfying (i) and (ii) of 3.6. Let $W_{j} \rightarrow U_{j} \times S$ be the pullback, under $\varphi_{j} \times \Phi: U_{j} \times S \rightarrow \mathbf{A}^{1} \times \mathbf{A}^{n+4 g}$, of

$$
\left[\operatorname{Spec} A[x, z] /\left(z^{m_{j}}-x\left(x-t_{j}\right)^{m_{j}-1}\right)\right]^{\amalg N / m_{j}}
$$

for $j \leqslant n$, and of

$$
\left[\operatorname{Spec} A[x, z] /\left(z^{m_{j}}-\left(x-\tau_{j}\right)\left(x-t_{j}\right)^{m_{j}-1}\right)\right]^{N / m_{j}}
$$


for $j>n$. Suppose $W$ and $W_{j}$ agree over an étale neighborhood of $\left(x_{j}, s\right)$. Then $W \rightarrow X \times H$ is an (algebraic) Hurwitz family for $(X ; x)$ with data $d$.

That such a family exists, and is essentially unique, will follow from Theorem 4.2.

4. A deformation theorem. In this section we prove a deformation theorem for covers of smooth proper $k$-schemes. Namely, suppose given a cover $Z \rightarrow X$, a Zariski open covering $\left\{U_{j}\right\}$ whose pairwise intersections avoid the branch locus, and a collection of covers $W_{j} \rightarrow U_{j} \times S$ which locally deform $Z \rightarrow X$. Then there exists an essentially unique cover $W \rightarrow X \times H$ which deforms $Z \rightarrow X$ and agrees with each $W_{j}$ locally in the étale topology.

The main ingredient in the proof is Artin's Algebraization Theorem [1]. Let $k$ be a field or an excellent Dedekind domain (e.g. a complete discrete valuation ring). Let $S$ be a scheme which is locally of finite type over $k$, and $F$ a contravariant functor from $S$-schemes to sets. If $T=\operatorname{Spec} B$ is an affine $S$-scheme, write $F(B)$ for $F(T)$. Assume that $F$ is limit preserving, i.e. $F$ commutes with direct limits on the ring level. Let $\bar{B}$ be a complete noetherian local $\Theta_{S}$-algebra.

Theorem 4.1 (Artin's Algebraization Theorem). Let $\xi_{0} \in F(k)$ and let $\hat{\xi} \in$ $F(\bar{B})$ be a deformation of $\xi_{0}$. Suppose that the formal deformation $\bar{\xi}$ corresponding to $\hat{\xi}$ is versal.

(i) $\bar{\xi}$ is algebraizable; i.e. there is a deformation $\xi \in F(T)$ of $\bar{\xi}$, for some $S$-scheme $T$ of finite type (with $\xi_{0}$ corresponding to a point $t$ of $T$ );

(ii) If $\bar{\xi}$ determines $\hat{\xi}$ uniquely, then $\xi$ extends $\hat{\xi}$, and $\xi$ is unique up to pullback by an étale neighborhood of $(T, t)$;

(iii) If $\bar{B}$ is of the form $\hat{\theta}_{Y, y}$, for $Y$ an $S$-scheme of finite type, then $(T, t)$ in (i) may be taken to be an étale neighborhood of $(Y, y)$.

Proof. Parts (i) and (ii) are Theorems 1.6 and 1.7 of [1], and part (iii) follows from the remark after the statement of Theorem 1.7.

Let $k$ be as above, and $Z \stackrel{\pi}{\rightarrow} X$ a cover of a smooth noetherian proper $k$-scheme. Let $\left\{U_{j}\right\}$ be an open covering of $X$, such that $Z$ is unramified over $U_{i} \cap U_{j}$, for $i \neq j$. Let $(S, s)$ be a pointed $k$-scheme of finite type, and $W_{j} \rightarrow U_{j} \times S$ be covers with branch locus $D_{j}$. Let $D$ be the closure in $X \times S$ of the union of the $D_{j}$ 's. Suppose that the fibre of $W_{j}$ over $U_{j} \times\{s\}$ is isomorphic, as a cover, to $Z_{U_{j}} \rightarrow U_{j}$. We then have the following deformation theorem:

THEOREM 4.2. Under the above hypotheses, there is an étale neighborhood $(H, h)$ of $(S, s)$ and a cover $W \rightarrow X \times H$ branched only over the pullback of $D$, such that

(i) the fibre of $W$ over $h$ is isomorphic to $Z \rightarrow X$; and

(ii) $W_{j}$ and $W$ agree in an étale neighborhood of $(X \times S,(x, s))$, for any $x \in U_{j}$.

Moreover, any two such $W \rightarrow X \times H$ agree over some common étale neighborhood of the $(H, h)$ 's.

Proof. We will first prove the theorem with (ii) replaced by the weaker

(ii) $)^{\prime}$ The pullback of $W_{j}$ to a cover $W_{j}^{\prime} \rightarrow U_{j} \times H$ agrees with $W_{U_{j}} \rightarrow U_{j} \times H$ over the formal completion at $U_{j} \times\{h\}$. 
The proof divides into several steps:

Step 1. $W$ exists infinitesimally, near $X \times\{s\}$.

We may assume that $S$ is affine; say $S=\operatorname{Spec} A$. For $Y \subset X$, let $Y_{r}=Y \times$ $\operatorname{Spec}\left(\Theta_{S, s} / \mathrm{m}_{s}^{r}\right)$ and let $\hat{Y}$ be the formal completion of $Y \times S$ at $Y \times\{s\}$. Similarly denote fibres over $Y_{r}$ and $\hat{Y}$. Write $Q=\pi_{*} \theta_{Z}, Q_{j, r}=\pi_{*} \theta_{W_{j, r}}, \hat{Q}_{j}=\pi_{*} \theta_{\hat{w}_{j}}$. We claim that there is a unique sheaf $\mathcal{Q}_{r}$ of $\hat{\theta}_{X_{r}}$-algebras such that $\mathbb{Q}_{r} \otimes \Theta_{X}=\mathscr{Q}$ and $\mathbb{Q}_{r} \mid U_{j, r}=\mathbb{Q}_{j, r}$. To see this, observe first that the affine open subsets of the $U_{j}$ 's form a basis for the topology of $X$. Thus defining $Q_{r}$ is equivalent to defining compatible isomorphisms $\mathbb{Q}_{i, r}\left(V_{r}\right) \rightarrow \mathbb{Q}_{j, r}\left(V_{r}\right)$ for $V \subset U_{i} \cap U_{j}$, whose pullbacks to the closed fibre are the transition functions for $\mathcal{Q}$. Now the spectra of $\mathcal{Q}_{i, r}\left(V_{r}\right)$ and $\mathcal{Q}_{j, r}\left(V_{r}\right)$ are étale over $V_{r}$ and thus are pullbacks of étale covers of $V\left(V_{r} \rightarrow V\right.$ being radicial). Hence the compatible isomorphisms can and must be defined by

$$
\mathbb{Q}_{i, r}\left(V_{r}\right) \leftarrow \mathbb{Q}(V) \otimes \mathcal{O}_{X_{r}} \stackrel{\sim}{\rightarrow} \mathbb{Q}_{j, r}\left(V_{r}\right) .
$$

This proves the claim and defines a covering $Z_{r} \rightarrow X_{r}$ satisfying (i) and (ii)' infinitesimally.

The above reasoning also applies to an arbitrary Artin local $k$-algebra $B$. Namely, suppose that $\theta_{S, s} \rightarrow B$ is an algebra homomorphism, defining a morphism of pointed schemes $(T, t) \stackrel{\varphi}{\rightarrow}(S, s)$, factoring through some $\operatorname{Spec}\left(\theta_{S, s} / \mathrm{m}^{r}\right)$. Then there is a unique cover $Z_{T} \rightarrow X \times T$ satisfying (i) and (ii)', namely the pullback $\varphi^{*} Z_{r}$.

Step 2. $W$ exists over a complete local neighborhood of $s$ in $S$, satisfying (i) and (ii)'.

Let $\hat{\mathscr{Q}}=\operatorname{proj} \lim \mathbb{Q}_{r}$, which makes sense because the $\mathcal{Q}_{r}$ 's are unique and hence compatible. This is the unique sheaf of $\vartheta_{\hat{X}}$-algebras such that $\hat{\mathscr{Q}} \otimes \vartheta_{X}=\mathscr{Q}$ and $\hat{\mathbb{Q}} \mid \hat{U}_{j}=\hat{Q}_{j}$. Since $X$ is proper, Grothendieck's Existence Theorem $[6,5.4 .5]$ shows that there is a unique coherent $\Theta_{X^{\prime}}$-algebra $\mathcal{Q}^{\prime}$ extending $\hat{\mathcal{Q}}$, where $X^{\prime}=X \times$ Spec $\hat{\theta}_{S, s}$. This defines a scheme $Z^{\prime}=$ Spec $_{\theta_{X}} Q^{\prime}$ which is finite over $X^{\prime}$.

We next show that $Z^{\prime} \rightarrow X^{\prime}$ is unramified away from $D \cap X^{\prime}$. Any irreducible component $C$ of the branch locus must meet the fibre $X \times\{s\}$ since $X$ is proper over $k$. So $C$ intersects some $\check{U}_{j}=\operatorname{Spec} \theta_{\hat{x}}\left(\hat{U}_{j}\right)$, and $C \cap \check{U} \subset D_{j} \cap \check{V}_{j}$. This implies that $C \subset D$. Thus the branch locus is as claimed, and $Z^{\prime} \rightarrow X^{\prime}$ is generically étale, hence a cover.

Step 3. $W$ exists satisfying the theorem, with (ii)' replacing (ii).

Define the following functor $F$ from $S$-schemes to sets: $F(T)$ is the set of isomorphism classes of pairs $(W \rightarrow X \times T, t)$, for which $W \rightarrow X \times T$ is a connected cover, $t \in T$ lies over the base point $s \in S$, the branch locus is the pullback of $D$, and conditions (i) and (ii) ${ }^{\prime}$ are met. The cover $Z^{\prime} \rightarrow X^{\prime}$ in Step 2 defines an element of $F\left(\hat{\theta}_{S, s}\right)$ which is an effective deformation of $Z \rightarrow X$, and every infinitesimal deformation has been shown to factor through it. On the other hand, a morphism $(T, t) \rightarrow(S, s)$ factors at most one way through $\operatorname{Spec} \mathcal{\theta}_{S, s} \hookrightarrow S$. Thus this effective deformation is universal (as a formal deformation).

To check that $F$ is limit preserving, let $\left\{B_{i}\right\}_{i \in I}$ be a filtered direct system of $A$-algebras, and $B=$ ind $\lim B_{i}$. We want to show that $F(B)=$ ind $\lim F\left(B_{i}\right)$. So let 
$T=\operatorname{Spec} B$, and $(W \rightarrow X \times T, t)$ be an element of $F(B)$. Since $\pi_{*} \vartheta_{W}$ is a coherent $\Theta_{X \times T}$-module, we may write

$$
\bigoplus \vartheta_{X \times T}\left(-n^{\prime}\right) \rightarrow \bigoplus \vartheta_{X \times T}(-n) \rightarrow \pi_{*} \vartheta_{W} \rightarrow 0 .
$$

If $U=\operatorname{Spec} R \subset X$ is a member of a finite affine Zariski open covering of $X$, then the left-hand arrow above is given locally over $U \times T$ by a matrix over $R \otimes B$. Since only finitely many elements are involved, it follows that the sheaf $\pi_{*} \theta_{W}$ is actually defined over some $B_{i}$, i.e. $W$ is the pullback of an element of some $F\left(B_{i}\right)$.

Thus the hypotheses of Artin's theorem are satisfied. Let $(W \rightarrow X \times H, h)$ be the advertised algebraization, with $(H, h)$ an étale neighborhood of $(S, s)$ which may be assumed connected. By construction $W \rightarrow X \times H$ has the correct branch locus, and satisfies (i) and (ii)' of the theorem. That it is essentially unique follows from 4.1(ii), whose hypothesis is satisfied because of Grothendieck's Existence Theorem.

Step 4. $W$ satisfies (ii).

Let $W \rightarrow X \times H$ be as in Step 3, and $x$ a point of $U_{j} \subset X$. We have already seen that $W$ and $W_{j}$ agree over $\check{U}_{j}$, so they agree over the formal completion $\widehat{X \times S}$ of $X \times S$ at $(x, s)$. That is, if $X \times S$ is identified with the formal completion of $X \times H$ at $(x, h)$, then there is a (unique) isomorphism $\varphi$ between the fibres of $W$ and $W_{j}$ over $U_{j} \times S$ which extends the isomorphism between the fibres over $U_{j} \times\{s\}$. Moreover, the same is true if $\widehat{X \times S}$ is replaced by any infinitesimal neighborhood $N \rightarrow X \times S$ of $(x, s)$, in which case the isomorphism for $N$ is the pullback of that for $\widehat{X \times S}$.

So let $S_{j}=U_{j} \times{ }_{k} S$, and define the contravariant functor $I$ from $S_{j}$-schemes to sets, sending $T$ to the set of isomorphisms of covers $W_{T} \rightarrow\left(W_{j}\right)_{T}$. The previous paragraph shows that the isomorphism $\varphi$ above is a universal effective deformation. Reasoning as in Step 3, the functor $I$ is limit preserving. The algebraization theorem then yields an étale neighborhood of $(x, s)$ in $X \times S$ over which $W$ and $W_{j}$ agree.

REMARK 4.3. Theorem 4.2 carries over from the category of covers to that of principal $G$-covers. Namely, the functor $F$ in Step 3 of the proof is altered by demanding that $W$ be equipped with a $G$-action which extends that of $Z \rightarrow X$ and is compatible with those of the $W_{j}$ 's in an étale neighborhood of each $(x, s)$. It then yields an algebraic family $W \rightarrow X \times H$ with these properties.

REMARK 4.4. Under the hypotheses of the theorem, suppose that each $W_{j}$ is ramified precisely over $D_{j}$. Let $D_{j}^{*}$ be the pullback of $D_{j}$ to $X \times H$, and let $D_{j}^{\prime}$ be the union of the connected components of $D_{j}^{*}$ meeting $U_{j} \times\{h\}$. Then the above proof shows that $W$ is branched precisely over the closure of the union of the $D_{j}^{\prime}$ 's. In particular, assume that $X$ is a curve and $D_{j}$ meets $X \times\{s\}$ transversally. Since $D_{j}^{\prime} \rightarrow H$ is proper, it has a well-defined degree, namely 1 . Thus each fibre $Z_{1} \rightarrow X \times\left\{h_{i}\right\}$ of $W$ is branched over the same number of points, counting multiplicity.

5. Algebraic Hurwitz families and the construction of covers. The existence of algebraic Hurwitz families with prescribed tame data can now be shown. Let $k$ be a 
field (resp. a complete discrete valuation ring), and let $X$ and $d \in G^{n+2 g}$ be as in 3.5 .

Proposition 5.1. There is an algebraic Hurwitz family for $(X, x)$ with data d. This family may be taken to be proper over $k$ if the characteristic (resp. the residue field characteristic) does not divide the order of $G$.

Proof. By Proposition 3.4 there is a locally standard mock cover $Z_{0} \rightarrow X$ of $(X, x)$ with data $d$. Let $W_{j} \rightarrow U_{j} \times S$ be as in 3.8. Theorem 4.2 provides a corresponding cover $W \rightarrow X \times H$, where $(H, h)$ is an étale neighborhood of $(S, s)$. The conclusions of the deformation theorem show that $W \rightarrow X \times H$ is a Hurwitz family with the desired data (under the $G$-action following from Remark 4.3).

If the order of $G$ is prime to the relevant characteristic, then let $\bar{H}$ be the normalization of $X^{n+4 g}$ in $H$, and let $\bar{W}$ be the normalization of $X \times \bar{H}$ in $W$. Both $\bar{H}$ and $W$ are proper over $k$, since $X$ is, and $W$ satisfies 3.6(i). As the ramification index over any component of $X \times(\bar{H}-H)$ must divide the order of $G$, all such ramification is tame. Abhyankar's Lemma implies that there is a cover $H^{\prime} \rightarrow \bar{H}$ whose pullback $V^{\prime} \rightarrow X \times H^{\prime}$ has the correct branch locus, and thus is a complete Hurwitz family with data $d$.

In the case that $k=\mathbf{C}$, this is indeed the algebraization of the construction of $\S 2$, by the following analog of [5, Proposition 7.3]:

Proposition 5.2. If $k=\mathrm{C}$, then the normalization of any complete algebraic Hurwitz family for $(X, x)$ with data $d$ is, under the complex topology, an analytic Hurwitz family for $(X, x)$ with data $d$.

Proof. Let $W^{\prime} \rightarrow X \times H^{\prime}$ be the normalization of the given algebraic Hurwitz family, and $H$ the analytic Hurwitz space. Let $H_{0}^{\prime}, H_{0}$ be their respective fibres over $X^{n+4 g}-\Delta$. As every algebraic cover is also an analytic one, a function $f_{0}$ : $H_{0}^{\prime} \rightarrow H_{0}$ is defined, with the property that for $h \in H_{0}^{\prime}$, the fibre $W_{h}^{\prime}$ is the cover corresponding to $f_{0}(h) \in H_{0}$. This function is continuous, by the obvious generalization of [5, Lemma 1.7], and is in fact analytic, because of the diagram:

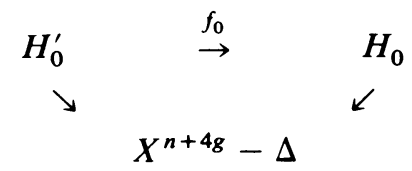

This map between covers extends to an $f: H^{\prime} \rightarrow H$, over $X^{n+4 g}$. Thus $W^{\prime} \rightarrow X \times$ $H^{\prime}$ is a component of an analytic Hurwitz family.

For $1 \leqslant j \leqslant n+2 g$, let $X_{j}$ be the curve in $X^{n+4 g}$ passing through the base point $s$ such that all coordinates but the $j$ th are held fixed. Let $H_{j}$ be the connected component of the base point $h \in H$ in the fibre over $X_{j}$, and let $W_{j}$ be the fibre over $H_{j}$. Apply the uniqueness part of Theorem 4.2 to $W_{j} \rightarrow X \times H_{j}$ and to $W^{\prime} \times{ }_{\mathbf{A}^{\prime}} H_{j} \rightarrow X \times H_{j}$, where $W^{\prime}$ is as in Example 3.7(b). This shows that $W_{j}$ is an analytic Hurwitz family of $\left(X ; x_{j}\right)$ with data $d_{j}$, its fibres over $t_{j}=y_{j}$ being the slit covers of $\left(X ; x_{j} ; y_{j}\right)$ with data $d_{j}$. Hence for $y=\left(y_{1}, \ldots, y_{n+2 g}\right)$ sufficiently close to $x=\left(x_{1}, \ldots, x_{n+2 g}\right)$, the normalized fibre of $W$ at a point of $H$ over 
$\left(x_{n+1}, \ldots, x_{n+2 g}, y_{1}, \ldots, y_{n+2 g}\right)$ is a slit cover of $(X ; x ; y)$ with data $d$. This implies the result.

Corollary 5.3. Let $Z \rightarrow X$ be a Galois $G$-cover of a Riemann surface $X$ of genus $g$, branched at $L=\left\{x_{0}, \ldots, x_{n}\right\}$. Let $d \in G^{n+2 g}$ be the corresponding data, relative to a standard homotopy basis for $X-L$, and $x_{n+1}, \ldots, x_{n+2 g}$ any points in $X-L$. Then any complete algebraic Hurwitz family for $\left(X ; x_{1}, \ldots, x_{n+2 g}\right)$ with data $d$ contains $Z \rightarrow X$ as the normalization of a fibre over $\left(x_{0}, \ldots, x_{0}\right)$.

Proof. By 5.2 and 2.5 .

Suppose now that $k$ is an algebraically closed field of characteristic $p \geqslant 0$, and that $X$ is a curve over $k$ of genus $g$. We preserve the notation of $\S 3.5$. Corollary 5.3 then generalizes, providing the promised construction of covers:

THEOREM 5.4. Let $d_{1}, \ldots, d_{n}$ be elements of $G$, a group of order prime to $p$. There is a bijection between

(i) Galois $G$-covers of $X$, branched at $L=\left\{x_{0}, \ldots, x_{n}\right\}$, whose inertial subgroups over $x_{j}$ are the conjugates of $d_{j}($ for all $j)$; and

(ii) $G$-covers of $X$ which occur as the normalization of irreducible fibres over $\left(x_{0}, \ldots, x_{0}\right) \in X^{n+4 g}$ in Hurwitz families with tame data $d=$ $\left(d_{1}, \ldots, d_{n}, \ldots, d_{n+2 g}\right) \in G^{n+2 g}$ such that the inertial subgroups over $x_{0}$ are the conjugates of $\left(d_{1} \cdots d_{n}\left[d_{n+1}, d_{n+g+1}\right] \cdots\left[d_{n+g}, d_{n+2 g}\right]\right)^{-1}$.

Proof. Let $W \rightarrow X \times H$ be a Hurwitz family as in (ii), with projection map $H \stackrel{\pi}{\rightarrow} X^{n+4 g}$. Let $H_{j} \subset H$ be the open subset $\pi^{-1}\left(\left(X-\left\{x_{j}\right\}\right)^{n+4 g}\right)$. Then 3.8 implies that for $h \in H_{j}$, the inertial subgroups in $W_{h}$ over $x_{j}$ are the conjugates of $d_{j}$. Thus the $G$-covers occurring in (ii) have the correct inertial subgroups over $x_{1}, \ldots, x_{n}$ and hence occur in (i) (being connected and hence Galois).

The converse, in the case $k=\mathbf{C}$, follows from Corollary 5.3. For general $k$, we proceed in a manner similar to the proof of [7, XIII, Corollaire 2.12]:

Case I. Char $k=0$. Let $Z \rightarrow X$ be a cover as in (i). There is a subfield $k_{0}$ of $k$, of finite transcendence degree over $\mathbf{Q}$, over which the cover and the $x_{j}$ 's are defined. Because of this finiteness, $k_{0}$ can be embedded in C. Let $k^{\prime}$ be an algebraically closed extension of $k_{0}$ for which there are $k_{0}$-morphisms of $k$ and of $\mathbf{C}$ into $k^{\prime}$. By [7, XIII, 1.14 and 2.9], the specialization morphisms from $k$ to $k^{\prime}$, and from $\mathbf{C}$ to $k^{\prime}$, are isomorphisms. Hence we may consider $Z \rightarrow X$ as a cover over $C$. As such it has data, say $d=\left(d_{1}, \ldots, d_{n+2 g}\right)$, relative to some standard homotopy basis of $X-L$ over C. Let $W \rightarrow X \times H$ be a complete Hurwitz family for $(X, x)$ over $k$ with this data. Again, there is an algebraically closed subfield $k^{\prime \prime}$ of $k$ over which the cover, the Hurwitz family, and their branch loci are defined, and which includes into $\mathbf{C}$. By the same reasoning as before, $W \rightarrow X \times H$ may be regarded as a cover over $\mathbf{C}$. As such, it is a Hurwitz family with data $d$, and so contains $Z \rightarrow X$ as a normalized fibre at a point $h \in H$ over $\left(x_{0}, \ldots, x_{0}\right)$, by Corollary 5.3. by the assumptions on $k^{\prime \prime}, h$ is a $k^{\prime \prime}$-valued point of $H$. By the specialization isomorphisms, the fibre over the corresponding $k$-valued point is the original $k$-valued cover $Z \rightarrow X$.

Case II. Char $k=p \neq 0$. There is a complete discrete valuation ring $R$ with 
residue field $k$ and fraction field $K$ of characteristic 0 . The ring $R$ contains elements $1 / n$ and $\zeta_{n}$ for $p \nmid n$, by Hensel's Lemma [10, p. 230]. By [7, III, 7.4], the curve $X$ is the reduction of a complete smooth curve $X_{R}$ over $R$. Let $\bar{K}$ be the algebraic closure of $K$, and $X_{\bar{K}}$ the fibre over $\bar{K}$.

Let $Z \rightarrow X$ be a cover as in (i). The appropriate specialization morphism from $k$ to $\bar{K}$ is an isomorphism [7, XIII, 1.14 and 2.9] because $p \nmid \# G$, so that $Z \rightarrow X$ corresponds to a cover $Z_{\bar{K}} \rightarrow X_{\bar{K}}$ over $\bar{K}$. By Case I, the latter cover occurs as a normalized fibre over $\left(x_{0}, \ldots, x_{0}\right)$ in a Hurwitz family for $(X, x)$ over $\bar{K}$, with some data $d$. Let $W_{R} \rightarrow X_{R} \times H_{R}$ be a complete Hurwitz family over $R$ with data $d$; this exists by Proposition 5.1. The fibre over $k$ is a Hurwitz family over $k$ with data $d$, and similarly for $\bar{K}$. Since the specialization map is an isomorphism, the result follows.

Let $X$ and $L$ be as in 5.4. The set of isomorphism classes of pairs $(G, d)$, where $d \in G^{n+2 g}$ and $p \nmid \# G$, form an inverse system $S$ of groups which is isomorphic to the inverse system $C$ of Galois groups of pointed $p^{\prime}$-Galois covers of $U=X-L$. The Hurwitz family construction allows us to state such an isomorphism algebraically:

Corollary 5.5. Complete Hurwitz families $W_{d} \rightarrow X \times H_{d}$ for $(X, x)$ may be picked, one for each choice of $p^{\prime}$-data $(G, d)$, so that they form an inverse system, indexed by $S$. This done, there exist inverse systems of points $h_{d} \in H_{d}$ over $\left(x_{0}, \ldots, x_{0}\right)$ satisfying (ii) of 5.4. Any such inverse system defines an isomorphism between $S$ and $C$, and thus an isomorphism between $\pi_{1}^{p^{\prime}}(X-L)$ and the free profinite $p^{\prime}$-group on $n+2 g$ generators.

Proof. Linearly order the elements of $S$ so that $\left(G^{\prime}, d^{\prime}\right)$ precedes $(G, d)$ whenever $\left(G^{\prime}, d^{\prime}\right)$ is a quotient of $(G, d)$. Similarly order a set of choices of Hurwitz families, and pull back each $H_{d}$ in turn so that it dominates each $H_{d^{\prime}}$ with lesser index. This produces a compatible set of Hurwitz families, giving an inverse system, rigidified by the base point (on the identity sheet) of the mock fibre.

Inverse systems of points $h_{d}$ exist for $k=\mathrm{C}$ by Proposition 5.2 and the proof of Proposition 2.5-at the very least, there is one for each standard homotopy basis of $(U, u)$. The specialization morphisms invoked in the proof of 5.4 show that these also exist for arbitrary $k$. Finally, any such inverse system defines, for each $d$, an isomorphism between the finite systems $S_{d}=\left\{\left(G^{\prime}, d^{\prime}\right) \mid d^{\prime} \leqslant d\right\}$ and $C_{d}=\{$ the Galois group of $\left.\left(W_{d^{\prime}}\right)_{h^{\prime}} \rightarrow X \mid d^{\prime} \leqslant d\right\}$. This increasing set of isomorphisms defines an inclusion of inverse systems $S \rightarrow C$. This inclusion is actually a bijection, and hence an isomorphism, because for each $G$, the number of pairs $(G, d)$ in $S$ equals the number of $G$-Galois covers of $U$.

REMARK 5.6. If $G$ is an arbitrary finite group, and $d \in G^{n+2 g}$ is tame, then the Hurwitz family $W \rightarrow X \times H$ of 5.1 may still be completed to a proper morphism $\bar{W} \rightarrow X \times \bar{H}$. If this has only separable ramification, then [2, Theorem 1.9] may replace Abhyankar's Lemma in the proof of 5.1, yielding a complete Hurwitz family. The specialization morphism arising in Case II of the proof of 5.4 is still an inclusion, though not an isomorphism (by the results in [7] cited in the proof). The 
Hurwitz family would therefore contain, as a normalized fibre over $\left(x_{0}, \ldots, x_{0}\right)$, a cover as in (i) of 5.4. Unfortunately the completion of the original Hurwitz family will sometimes be inseparable over $\bar{H}-H$, and indeed not all characteristic 0 covers with $p^{\prime}$-ramification descend to characteristic $p$.

5.7. Another possible application of the above algebraic Hurwitz family construction is to the moduli problem for curves. Mumford suggests [11] that one approach to studying the moduli spaces $M_{g}$ for curves of genus $g$ might be to describe explicitly the Hurwitz family $W \rightarrow H^{N, r}$ of $N$-sheeted simple covers of $\mathbf{P}^{1}$ branched at $r$ points (cf. §2.3). This is because every curve of genus $g$ arises as a $(g+1)$-sheeted simple cover of $\mathbf{P}^{1}$ branched at $4 g$ points. Indeed, Fulton [5] uses this fact to show that $M_{g}$ is irreducible, knowing that $H^{N, r}$ is (provided $p \nmid N$ !). In our context, $W \rightarrow H^{N, r}$ is an $N$-sheeted, non-Galois quotient of a Hurwitz family with group $S_{N}$ (the symmetric group) and data

$$
((12),(13), \ldots,(1 N), \ldots,(1 N)) \text {. }
$$

Such a family exists by 5.1 . Alternatively, Theorem 4.2 may be applied directly to the $N$-sheeted simple mock cover whose sheets intersect in pairs over the various branch points, as indicated by (5.8). Either way, the resulting cover may be completed, as in 5.1, provided that $p \nmid N$ !. This gives Fulton's Hurwitz family of simple covers (or possibly a cover of it), whose parameter space is a cover of the completion of $M_{g}$.

6. An example. In this section a simple example is given to illustrate the algebraic construction of Hurwitz families and covers given above. Let $k$ be an algebraically closed field of characteristic $\neq 2,3$, let $\zeta=\zeta_{6}$ be a primitive sixth root of unity, and consider the points $x_{0}=1, x_{1}=0, x_{2}=\infty$ in $\mathbf{P}_{k}^{1}$. Let $G$ be the cyclic group of order 6, with generator $g$. We will construct the Hurwitz family for $\left(\mathbf{P}^{1} ; 0, \infty\right)$ with data $\left(g, g^{3}\right)$, and use it to construct the cover of $\mathbf{P}^{1}$, branched over the $x_{i}$ 's, with this data.

First, we construct the $G$-mock cover $Z_{0} \rightarrow \mathbf{P}^{1}$ branched at $\{0, \infty\}$, with data $\left(g, g^{3}\right)$, as in Proposition 3.4. Write $\mathbf{P}^{1}=U_{1} \cup U_{2}$, where $U_{1}=\operatorname{Spec} k[x], U_{2}=$ Spec $k[\bar{x}]$, and $x \bar{x}=1$ in $U_{1} \cap U_{2}$. Over $U_{1}, Z_{0}$ is given by $z^{6}=x$ and over $U_{2}$ by $w^{2}=\bar{x}^{2}, v^{3}=1$. Choosing the set $\left\{1, g^{2}, g^{4}\right\}$ of coset representatives of the subgroup generated by $g^{3}$, and $\{1\}$ for that generated by $g$, we find that on sheet $g^{i}$

Thus

$$
z=\zeta^{i} x ; \quad w=(-1)^{i} \bar{x}, \quad u=\zeta^{2 i}
$$

$$
z=x^{2} u^{2} w ; \quad w=\bar{x}^{4} z^{3}, \quad v=\bar{x}^{2} z^{2}
$$

Let $Z_{r}$ be the standard infinitesimal deformation of $Z_{0}$ over Spec $k\left[t_{1}, t_{2}\right] /\left(t_{1}, t_{2}\right)^{r}$. Over $\left(U_{1}\right)_{r}, Z_{r}$ is given by

$$
z^{6}=x\left(x-t_{1}\right)^{5}
$$

and over $\left(U_{2}\right)_{r}$ by

$$
w^{2}=\bar{x}\left(\bar{x}-t_{2}\right), \quad v^{3}=1
$$


The patching is uniquely determined by the condition that it extend that given in (6.1). Thus

$$
w\left(1-\bar{x} t_{1}\right)^{5 / 2}=\bar{x}^{4} z^{3}\left(1-x t_{2}\right)^{1 / 2} ; \quad v\left(1-\bar{x} t_{1}\right)^{1 / 3}=\bar{x}^{2} z^{2}
$$

where $\left(1-x t_{2}\right)^{1 / 2}=1-x t_{2} / 2+\cdots$ denotes the unique square root of $1-x t_{2}$ in $k\left[t_{1}, t_{2}, x\right] /\left(t^{r}\right)$ which is congruent to 1 modulo $\left(t_{1}, t_{2}\right)$, and similarly for the other radicals.

Expressions (6.2)-(6.4) defines a compatible system of infinitesimal deformations, and hence a formal deformation. But they do not directly define a deformation parametrized by Spec $k\left[\left[t_{1}, t_{2}\right]\right]$, because the radicals in (6.4) do not exist in $k\left[\left[t_{1}, t_{2}\right]\right][x]$. Alternatively, the problem is that the local sections $z, w, v$ do not define global sections over $\mathbf{P}^{1} \times \operatorname{Spf} k\left[\left[t_{1}, t_{2}\right]\right]$, even after arbitrarily high twisting. The proof of Grothendieck's Existence Theorem, though, shows that there are enough global sections to span the formal module, after some fixed amount of twisting. In this case these can be taken to be

$$
z_{1}=z\left(1-x t_{2}\right)^{1 / 2}, \quad z_{2}=z^{2}
$$

on $U_{1}$, and

$$
\bar{z}_{1}=w v^{2}\left(1-\bar{x} t_{1}\right)^{5 / 6}, \quad \bar{z}_{2}=v\left(1-\bar{x} t_{1}\right)^{5 / 3}
$$

on $U_{2}$. These local sections exist in the formal module, because the radicals exist in $k[x]\left[\left[t_{1}, t_{2}\right]\right]$ and $k[\bar{x}]\left[\left[t_{1}, t_{2}\right]\right]$. Over $U_{1}, z_{1}$ and $z_{2}$ generate the formal module because $\left(1-x t_{2}\right)^{1 / 2}$ is a unit; similarly $\bar{z}_{1}$ and $\bar{z}_{2}$ generate over $U_{2}$, observing that $\bar{z}_{1} \bar{z}_{2}=w\left(1-\bar{x} t_{1}\right)^{5 / 2}$. These sections satisfy

$$
z_{1}^{6}=x\left(x-t_{1}\right)^{5}\left(1-x t_{2}\right)^{3}, \quad z_{2}^{3}=x\left(x-t_{1}\right)^{5}
$$

and

$$
\bar{z}_{1}^{6}=\bar{x}^{3}\left(1-\bar{x} t_{1}\right)^{5}\left(\bar{x}-t_{2}\right)^{3}, \quad \bar{z}_{2}^{3}=\left(1-\bar{x} t_{1}\right)^{5}
$$

with patching given by

$$
\bar{z}_{1}=\bar{x}^{2} z_{1}, \quad \bar{z}_{2}=\bar{x}^{2} z_{2}
$$

Expressions (6.7)-(6.9) also define an (effective) deformation over $k\left[\left[t_{1}, t_{2}\right]\right]$, and indeed an algebraic deformation over $k\left[t_{1}, t_{2}\right]$. (Observe that the original sections $z, v, w$ no longer exist.) A given fibre is branched at $x=0, \infty, t_{1}, 1 / t_{2}$, and the fibre over $\left(t_{1}=1, t_{2}=1\right)$ is given over $U_{1}$ by

$$
z_{1}^{6}=-x(x-1)^{8}, \quad z_{2}^{3}=x(x-1)^{5} .
$$

Its normalization, which is the normalization of

$$
z^{6}=x(x-1)^{2}
$$

is the desired cover.

M. Fried suggests that the algebraic construction of Hurwitz families may yield an algorithm providing calculations as above for arbitrary tame group data. Such an algorithm would give explicit equations for all tamely ramified covers of curves, and presumably also for moduli spaces for curves (cf. §5.7). This possibility seems to rest upon whether Grothendieck's Existence Theorem and Artin's Algebraization Theorem are "algorithmic" in nature. 


\section{REFERENCES}

1. M. Artin, Algebraization of formal moduli. I, Global Analysis: Papers in Honor of K. Kodaira, Princeton Univ. Press, Princeton, N. J., 1969, pp. 21-71.

2. H. Epp, Eliminating wild ramification, Invent. Math. 19 (1973), 235-249.

3. M. Fried, Field of definition of function fields and Hurwitz families, and groups as Galois groups, Comm. Algebra 5 (1977), 17-82.

4.___ Galois groups and complex multiplication, Trans. Amer. Math. Soc. 235 (1978), 141-163.

5. W. Fulton, Hurwitz schemes and irreducibility of moduli of algebraic curves, Ann. of Math. (2) 90 (1969), 543-573.

6. A. Grothendieck and J. Dieudonné, Eléments de géométrie algébrique. III (EGA III), part 1, Publ. Math. Inst. Hautes Études Scientifiques 11 (1961).

7._Séminaire de geométrie algébrique. 1 (SGA 1). Revêtments étales et groupe fondamental, Lecture Notes in Math., vol. 224, Springer, Berlin, 1971.

8. D. Harbater, Deformation theory and the fundamental group in algebraic geometry, Ph.D. Thesis, Mass. Inst. Tech., Cambridge, Mass., 1978.

9. A. Hurwitz, Ueber Riemann'sche Flächen mit gegebenen Verzweigungspunkten, Math. Ann. 39 (1891), 1-61.

10. N. Jacobson, Lectures in abstract algebra. III, Van Nostrand, Princeton, N. J., 1964.

11. D. Mumford, Problems in present day mathematics: algebraic geometry, in Mathematical Developments Arising from Hilbert Problems, Proc. Sympos. Pure Math., vol. 28, Amer. Math. Soc., Providence, R. I., 1976, pp. 44-45.

Department of Mathematics, Harvard University, Cambridge, Massachusetts, 02138

Department of Mathematics, University of Pennsylvania, Philadelphia, Pennsylvania, 19104 (Current address) 